\title{
Self-Consistent Calculation on the Time-Dependent Electrons Transport Properties of a Quantum Wire
}

\author{
J. Chuen, D. Y. Li, Y. Tian, and L. X. Shao \\ School of Physics Science and Technology, Lingnan Normal University, Zhanjiang 524048, China \\ Correspondence should be addressed to L. X. Shao; phy_quan@foxmail.com
}

Received 27 March 2015; Revised 29 June 2015; Accepted 30 June 2015

Academic Editor: Ottorino Ori

Copyright (C) 2015 J. Chuen et al. This is an open access article distributed under the Creative Commons Attribution License, which permits unrestricted use, distribution, and reproduction in any medium, provided the original work is properly cited.

\begin{abstract}
Responses of a quantum wire $(\mathrm{QW})$ connected with wide reservoirs to time-dependent external voltages are investigated in self-consistent manner. Distributions of the internal potential and the induced charge density, capacitance, and conductance are calculated. Results indicate that these physical quantities depend strongly on the Fermi energy of systems and the frequency of external voltages. With the increase of the Fermi energy, capacitance and conductance show some resonant peaks due to the open of the next higher quantum channels and the oscillations related to the longitudinal resonant electron states. Frequency-dependent conductance shows two different responses to the external voltages, inductive-like and capacitive-like; and the peaks structure of capacitance is related to the plasmon-like excitation in mesoscopic conductor.
\end{abstract}

\section{Introduction}

With the development of nanotechnology, it is possible to define constrictions with geometrical dimension which is smaller than the elastic, inelastic mean free paths and with various forms, for example, quantum wires (QWs). Therefore, electrons transport in these constriction structures has attracted great research interest in recent years, both experimentally and theoretically, because of its fundamental importance and potential microelectronic applications [1-6]. Studies of the responses of the QWs to external voltages have been frequently reported in the literatures. An important issue is the effect of the Coulomb interaction between electrons on the conductance of the QWs. For a time-dependent case, the interaction plays a key role in ensuring the charge conservation and the gauge invariance under a potential shift [1-3]. Gasparian et al. had used the linear response theory (LRT) and the scattering matrix theory (SMT) to develop a theoretical formalism to analyze the responses of mesoscopic conductors to external disturbance [7]. This is an important advance in the theory of mesoscopic physics: the first step in this theory is to consider the system responses to external perturbation; the second step is to consider the internal potential formed by the interaction. The effect of the interaction on the ac conductance of a QW with reservoirs was studied in
[8] by using the random phase approximation (RPA). Based on the Hartree-Fock approximation (HFA), the distributions of the internal potential and electron density in the system were investigated [9]. Also, Sablikov and Shchamkhalova studied the time-dependent electron transport in the QW with Coulomb interaction and gave the distributions of the internal potential and electron density [10]. Furthermore, the ac response in QWs with infinite length had been discussed [11]. Hence, the responses to the external voltages, for instance, the ac conductance, the distributions of the internal potential, and electron density in the QWs, have been investigated. But the interacting current response and internal potential were determined by some experiential approaches [2] or by Thomas-Fermi approximation (TFA) in the low-frequency limit [12]. However, the mesoscopic devices in future all work on the higher frequencies conditions; so some detailed knowledge of the time-dependent transport properties of mesoscopic structures are required. In our previous work [13], we have developed a general selfconsistent electrons dynamic transport theory for multiprobe mesoscopic conductor, in which $e$-e interaction is fully considered. In our theory, since the local current response, the charge induced by external voltages, and the Lindhard function are generally formulated, in principle, the internal potential and interacting conductance of any systems can 


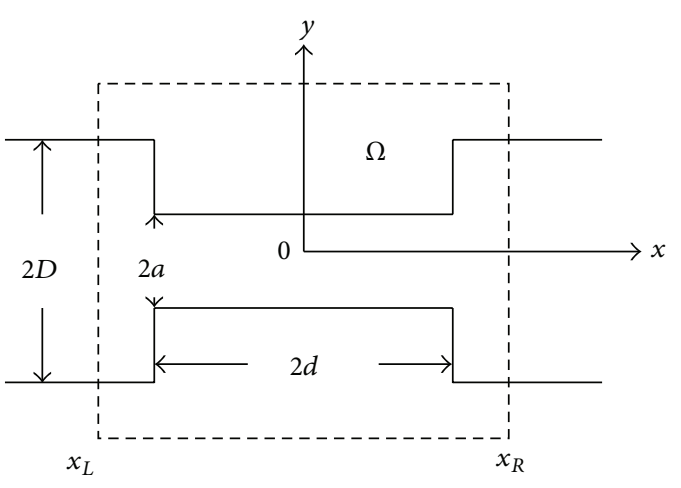

FIGURE 1: Schematic view of a quantum wire (QW) connected with two wide reservoirs. $x_{L}=-x_{R}$.

be calculated by self-consistent manner. In this paper, we will briefly review the self-consistent theory and calculate the internal characteristic potential and the induced charge density in a QW including the transition regions and present the numerical verification of the mesoscopic capacitance and conductance.

\section{Theory and Model}

In presence of time-dependent external voltages with frequency $\omega$, as a response of multiprobe mesoscopic conductor in shielding area $\Omega$ to the voltages, the internal characteristic potential function in $\alpha$-electrode (reservoir) can be obtained via Poisson equation [13]

$$
\begin{aligned}
& \Delta u_{\alpha}(\mathbf{r}, \omega)+\frac{1}{\varepsilon_{0}} \int_{\Omega} \Pi\left(\mathbf{r}, \mathbf{r}^{\prime}, \omega\right) u_{\alpha}\left(\mathbf{r}^{\prime}, \omega\right) \mathrm{d} \mathbf{r}^{\prime} \\
& \quad=-\frac{1}{\varepsilon_{0}} \rho_{\alpha}^{\operatorname{ext}}(\mathbf{r}, \omega)
\end{aligned}
$$

with $\Pi\left(\mathbf{r}, \mathbf{r}^{\prime}, \omega\right)$ representing the Lindhard function, and the term on the right hand side describes the charge density due to the voltages. $\varepsilon_{0}$ is the dielectric constant in a vacuum.

Below we consider a model that includes a narrow ballistic QW with width $2 a$, that is, QC (C: $|x| \leq d$ and $|y| \leq 2 a$ ), and two large electrons reservoirs with width $2 D$, that is, the left $(L: x<-d$ and $|y| \leq 2 D)$ and right $(R: x>d$ and $|y| \leq 2 D$ ) reservoirs, as shown in Figure 1 . The dashed line box labeled by $\Omega$ in Figure 1 includes the whole QW and parts of the reservoirs, and it is large enough to cover all the regions with varying distributions of potential and charge. This means that all the electric-field lines come from and end at the charge inside $\Omega$.

To proceed, we assume that the electrons with effective mass $m_{e}^{*}$ in the system are confined by hard-wall boundaries along $y$-direction but allowed to move along the $x$-direction; then the transverse eigenstates $\varphi_{l}(y)$ with eigenvalues $\varepsilon_{l}$ given by $\varepsilon_{l}=l^{2} \pi^{2} \hbar^{2} / 2 m_{e}^{*} w^{2}, l=1,2,3, \ldots$, with $w=\left\{\begin{array}{l}2 D|x| \geq d \\ 2 a|x| \leq d\end{array}\right.$
( $l$ refers to the transverse modes or channels number) in the system read

$$
\varphi_{l}(y)=\sqrt{\frac{2}{w}} \sin \frac{l \pi}{w}\left(y+\frac{w}{2}\right) .
$$

The complete waves with energy $E$, transverse state $l^{\prime}$, and longitudinal wave number $k_{\delta}^{l^{\prime}}=k_{\delta}^{l^{\prime}}(E)=\sqrt{2 m_{e}^{*}\left(E-\varepsilon_{l^{\prime}}\right)} / \hbar$ in $\alpha$-reservoir read

$$
\psi_{l}^{\alpha}(x, y)=\sum_{l^{\prime}} \widetilde{\chi}_{k_{\delta}^{l^{\prime}}}(x) \varphi_{l^{\prime}}(y)
$$

with

$$
\begin{aligned}
& \tilde{\chi}_{k_{\delta}^{l^{\prime}}}(x)=\delta_{l l^{\prime}} \delta_{\alpha \delta} \chi_{k_{\delta}^{l^{\prime}}}^{+}(x)+\left(\frac{v_{\alpha}^{l}}{v_{\delta}^{l^{\prime}}}\right)^{1 / 2} s_{\alpha \delta, l l^{\prime}} \chi_{k_{\delta}^{l^{\prime}}}^{-}(x), \\
& \chi_{k_{\delta}^{l^{\prime}}}^{\sigma}(x)=e^{i \sigma k_{\delta}^{l^{\prime}} x,} \\
& \sigma= \pm 1, \alpha, \delta=L, R,
\end{aligned}
$$

where $s_{\alpha \delta, l l^{\prime}}$ denotes the transmission amplitude for going from mode $l$ in lead $\alpha$ to mode $l^{\prime}$ in lead $\delta . v_{\alpha}^{l}=\hbar k_{\alpha}^{l} / m_{e}^{*}$ is the incident velocity of the electrons in the transport direction. $\sigma= \pm 1$ and indexes the incoming $(\sigma=+1)$ or outgoing $(\sigma=-1)$ waves from the left or right reservoir, respectively.

In the QW, the wave functions of electron may be expanded as

$$
\psi_{l}^{C}(x, y)=\sum_{m} \widetilde{\chi}_{k_{C}^{m}}(x) \varphi_{m}(y)
$$

with

$$
\tilde{\chi}_{k_{C}^{m}}(x)=\sum_{\sigma} A_{m l}^{\sigma} \chi_{k_{m}}^{\sigma}(x), \quad \sigma= \pm 1,
$$

where $A_{m l}^{\sigma}$ are the probability amplitudes of the waves.

The unknown coefficients in the above wave functions can be determined by using the boundary conditions of the wave functions. In general, $\psi_{l}^{C}(x, y)$ is now matched to $\psi_{l}^{\alpha}(x, y)$ at $x= \pm d$ with the requirement that amplitudes and derivatives with respect to $x$ are equal; then the scattering states wave functions of electrons in transition region may be eliminated.

2.1. Internal Characteristic Potential and Charge Density. Ignoring the fringe field effect and using the fact $\int\left|\varphi_{l(m)}(y)\right|^{2} \mathrm{~d} y \mathrm{~d} z=1$, (1) can be casted into

$$
\begin{aligned}
& \Delta u_{\alpha}(x, \omega)+\frac{1}{S \varepsilon_{0}} \int_{\Omega} \Pi\left(x, x^{\prime}, \omega\right) u_{\alpha}\left(x^{\prime}, \omega\right) \mathrm{d} x^{\prime} \\
& =-\frac{1}{S \varepsilon_{0}} \rho_{\alpha}^{\text {ext }}(x, \omega),
\end{aligned}
$$


where $S(S=w \times h), h$ are the cross-sectional area and thickness of the QW, respectively; and

$$
\begin{array}{r}
\operatorname{Im}\left[\Pi\left(x, x^{\prime}, \omega\right)\right] \\
=\frac{e^{2}}{2 \hbar} \sum_{\alpha, \beta, l} \int \frac{\varphi_{\alpha \beta}^{l l}(x) \varphi_{\beta_{\alpha}}^{l l}\left(x^{\prime}\right)}{v_{l} \widetilde{v}_{l}}\left(f_{l}-\widetilde{f}_{l}\right) \mathrm{d} E \\
\alpha, \beta=L, R, C
\end{array}
$$

with

$$
\begin{aligned}
& v_{l}=v_{l}(E)=\sqrt{\frac{2\left(E-\varepsilon_{l}\right)}{m_{e}^{*}}}, \\
& \varphi_{\alpha \beta}^{l l}(x)=\left[\widetilde{\chi}_{k_{\alpha}^{l}}(x)\right]^{*} \widetilde{\chi}_{k_{\beta}^{l}}(x), \\
& \operatorname{Im}\left[\rho_{\alpha}^{\text {ext }}(x, \omega)\right] \\
& =\frac{e^{2}}{2 \hbar^{2}} \sum_{\beta, l} \int \frac{\varphi_{\alpha \beta}^{l l}(x) I_{\beta \alpha}\left(E, \omega, x_{L}\right)}{v_{l} \widetilde{v}_{l}} \frac{f_{l}-\tilde{f}_{l}}{\hbar \omega} \mathrm{d} E
\end{aligned}
$$

with

$$
\begin{aligned}
& I_{\alpha \beta}\left(E, \omega, x_{L}\right) \\
& =\frac{\hbar}{2 i m_{e}^{*}}\left[\delta_{\alpha \beta} I_{\alpha \beta}^{++}\left(x_{L}\right)+S_{\alpha \beta, l l}^{\dagger}(E) S_{\alpha \beta, l l}(E) I_{\alpha \beta}^{--}\left(x_{L}\right)\right], \\
& I_{\alpha \beta}^{\sigma_{1} \sigma_{2}}=i\left(\sigma_{1} k_{\alpha}^{l}+\sigma_{2} k_{\beta}^{l}\right) e^{i\left(\sigma_{2} k_{\alpha}^{l}-\sigma_{1} k_{\beta}^{l}\right) x_{L}}, \\
& \qquad \sigma_{1}, \sigma_{2}= \pm 1, \sigma_{1} \cdot \sigma_{2}=1,
\end{aligned}
$$

where $f_{l}=f_{l}(E)$ is the Fermi-Dirac distribution functions and $\widetilde{f}=f(E+\hbar \omega)$ and $\widetilde{v}_{l}=v(E+\hbar \omega)$. Then, the real parts of $\rho_{\alpha}^{\text {ext }}(x, \omega)$ and $\Pi\left(x, x^{\prime}, \omega\right)$ can be obtained by applying the Kramers-Kronig relation

$$
\operatorname{Re}[F(x, \omega)]=\frac{2}{\pi} P \int \frac{v \operatorname{Im}[F(x, \omega)]}{v^{2}-\omega^{2}} \mathrm{~d} v .
$$

Hence, the total dynamic response of the induced charge density to the external voltages in the QW can be read [13]:

$$
\rho_{\alpha}(x, \omega)=\rho_{\alpha}^{\text {ext }}(x, \omega)+\int \Pi\left(x, x^{\prime}, \omega\right) u_{\alpha}\left(x^{\prime}, \omega\right) \mathrm{d} x^{\prime} .
$$

2.2. Mesoscopic Capacitance and Conductance. According to (12), when a small voltage $\delta V_{\alpha}(\omega)$ is applied to the $\alpha$-reservoir, the total charge $\delta Q_{\alpha}(\omega)$ in the half portion of $\Omega$ is given by

$$
\delta Q_{\alpha}(\omega)=S \int \rho_{\alpha}(x, \omega) \delta V_{\alpha}(\omega) \mathrm{d} x
$$

The capacitance of the system can be defined as

$$
C(\omega)=\frac{\delta Q_{\alpha}(\omega)}{\delta V_{\alpha}(\omega)} .
$$

Substituting (13) into (14), we have

$$
C(\omega)=S \int_{x_{L}}^{0} \rho_{L}(x, \omega) \mathrm{d} x=-S \int_{0}^{x_{R}} \rho_{R}(x, \omega) \mathrm{d} x .
$$

With Poisson equation,

$$
\rho_{\alpha}(x, \omega)=-\varepsilon_{0} S \frac{\partial^{2}}{\partial x^{2}} u_{\alpha}(x, \omega)
$$

we may then write, instead of (14),

$$
\begin{aligned}
C(\omega) & =-\left.\varepsilon_{0} S \frac{\partial}{\partial x} u_{L}(x, \omega)\right|_{x=x_{L}} \\
& =\left.\varepsilon_{0} S \frac{\partial}{\partial x} u_{R}(x, \omega)\right|_{x=x_{R}} .
\end{aligned}
$$

For the mesoscopic devices, the contribution to the total conductance consists of the flow of charged particles through the terminal and the current due to the interaction. Also, the particles conductance can be read directly from [13]

$$
\begin{aligned}
& \operatorname{Re}\left[G_{\alpha \beta}^{\text {ext }}(\omega)\right] \\
& =\frac{e^{2}}{2 \hbar} \sum_{l} \int \frac{I_{\alpha \beta}\left(E, \omega, x_{L}\right) I_{\beta \alpha}\left(E, \omega, x_{L}\right)}{v_{l} \widetilde{v}_{l}} \frac{f_{l}-f_{l}^{+}}{\omega} \mathrm{d} E \\
& \alpha, \beta=L, R, \\
& \operatorname{Im}\left[G_{\alpha \beta}^{\text {ext }}(\omega)\right]=-\frac{2 \omega}{\pi} P \int \frac{\operatorname{Re}\left[G_{\alpha \beta}^{\text {ext }}(\omega)\right]}{v^{2}-\omega^{2}} \mathrm{~d} v .
\end{aligned}
$$

As pointed above all, in order to current conserve, the current due to the charge accumulation in the scattering region has to be taken into account. This implies that, for each lead $\alpha$, the sum of the current coming from all leads equals zero; that is, $\sum_{\alpha} I_{\alpha}=0$. Now we rewrite the current conservation equation as a Kirchhoff rule: $\sum_{\alpha} I_{\alpha}^{\text {ext }}+I^{\text {int }}=$ 0 , where $I_{\alpha}^{\text {ext }}$ and $I^{\text {int }}$ are the currents due to the external fields (voltages) and the internal self-consistent potential $U$, respectively. After due consideration of the external fields and the self-consistent internal potential in the frequency domain we have [1]

$$
\begin{aligned}
I_{\alpha}(\omega) & =\sum_{\beta} G_{\alpha \beta}^{\mathrm{ext}}(\omega) \delta V_{\beta}(\omega)+G_{\alpha}^{\mathrm{int}}(\omega) U(\omega) \\
& =\sum_{\beta} G_{\alpha \beta}(\omega) \delta V_{\beta}(\omega),
\end{aligned}
$$

where $G_{\alpha \beta}$ is the total conductance matrix of the mesoscopic conductor. To proceed, we assume that the ratio of the current $\left(I_{\alpha}^{\text {int }}\right)$ in lead $\alpha$ to $I^{\text {int }}$ is $P_{\alpha}$, which obeys $\sum_{\alpha} P_{\alpha}=1$. Then the total conductance can be given by [14]

$$
G_{\alpha \beta}(\omega)=G_{\alpha \beta}^{\mathrm{ext}}(\omega)+P_{\alpha} G_{\beta}^{\mathrm{int}}(\omega),
$$

where the second term on the right hand side is the internal conductance. 
Current conservation implies $\sum_{\alpha} I_{\alpha}(\omega)=0$; we obtain

$$
\begin{aligned}
U(\omega) & =-\frac{\sum_{\alpha \beta} G_{\alpha \beta}^{\mathrm{ext}}(\omega) V_{\beta}(\omega)}{\sum_{\alpha} G_{\alpha}^{\mathrm{int}}(\omega)}, \\
G_{\beta}^{\text {int }}(\omega) & =-\sum_{\alpha} G_{\alpha \beta}^{\mathrm{ext}}(\omega) .
\end{aligned}
$$

Substituting (21) back into (19), we have again that

$$
G_{\alpha \beta}(\omega)=G_{\alpha \beta}^{\mathrm{ext}}(\omega)-\frac{\sum_{\gamma} G_{\alpha \gamma}^{\mathrm{ext}}(\omega) \sum_{\delta} G_{\delta \beta}^{\mathrm{ext}}(\omega)}{\sum_{\delta \gamma} G_{\delta \gamma}^{\mathrm{ext}}(\omega)} .
$$

\section{Results and Discussions}

In numerical calculation, the length, energy, and conductance are measured in units of $a, \Delta=\pi^{2} \hbar^{2} / 2 m_{e}^{*} a^{2}$, and $e^{2} / 2 \hbar$, respectively; $a=50 \mathrm{~nm}, D=50$, and $d=5$. The frequency $\omega$ of voltage in the normalized value, that is, $\omega \rightarrow \hbar \omega / \Delta$. Moreover, to solve (7) for $u_{\alpha}(x, \omega)$, the following boundary condition to determine $u_{\alpha}(x, \omega)$ is adopted:

$$
u_{\alpha}(x, \omega)= \begin{cases}1 & \alpha=\beta \\ 0 & \alpha \neq \beta\end{cases}
$$

In Figures 2-4 we present the distributions of the internal characteristic potential function $u_{L}(x)$ and charge density $\rho_{L}(x)$ for two values of external frequency $\omega=1,2.8$ by setting the Fermi energy $E_{F}=0.8$, which is smaller than transverse ground energy of the QW. In this case, the transverse ground energy plays a key role of a potential barrier for the incident electrons, so the electrons show the behavior of single barrier tunneling; it is equivalent to a barrier capacitor. From the profiles of $u_{L}(x)$, one can find that $u_{L}(x)$ is a smooth curve and almost linear inside the transition region of the QW. Also, the potential drop is uniform between the two ends of the shielding area $\Omega$. Under the zero frequency, in comparison with the results given by the self-consistent manner (SC) with results of the Thomas-Fermi approximation (TFA), one can find that in this case our results agree well with that of the TFA, but, with the increase of the frequency, $u_{L}(x)$ shows some notable differences between them and the potential drop occurs not only in the two ends of reservoirs but also in the reservoirs.

The induced charge is considerable only around the left and right ends of the QW, and some small oscillation can be observed, as shown in Figures 3 and 4. Moreover, the charge otherwise is almost zero, but the variation of the characteristic potential does not vanish; this is because the one-dimensional charge distribution is given by integrating over the crosssectional area of the system, and the cross-sectional area of reservoir is much larger than that of the QW. Importantly, in comparison with results given by preceding researches, $u_{L}(x)$ and $\rho_{L}(x)$ are complex with extremely small imaginary part (here, the imaginary part of $u_{L}(x)$ is too small, so it isn't presented in Figure 2) and the imaginary part of them should not be ignored because it gives rise to a real admittance, which actually corresponds to the charge-relaxation resistance.

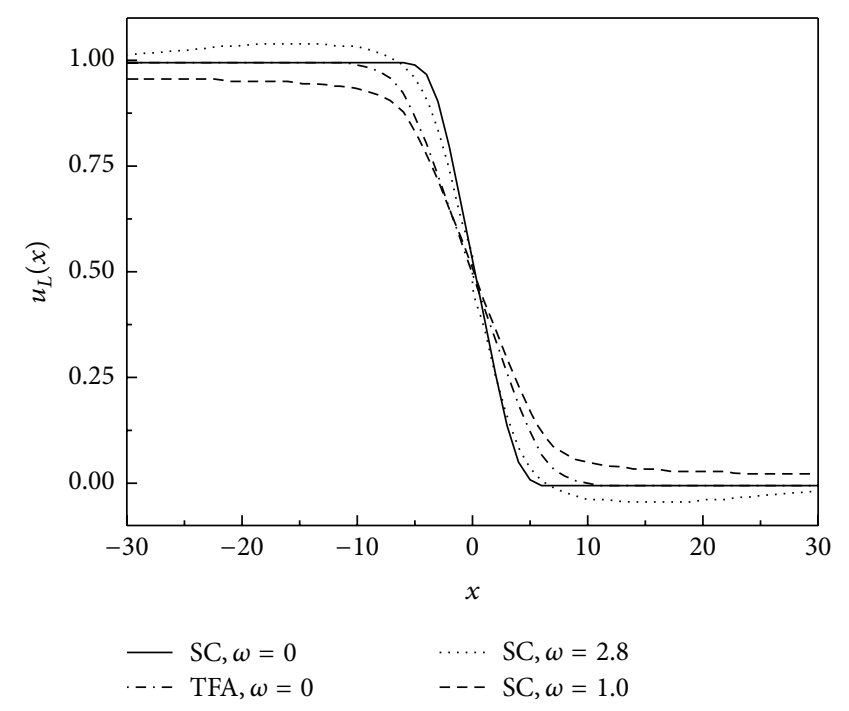

FIgURE 2: Distributions of the internal characteristic potential by setting $\omega=1,2.8$.

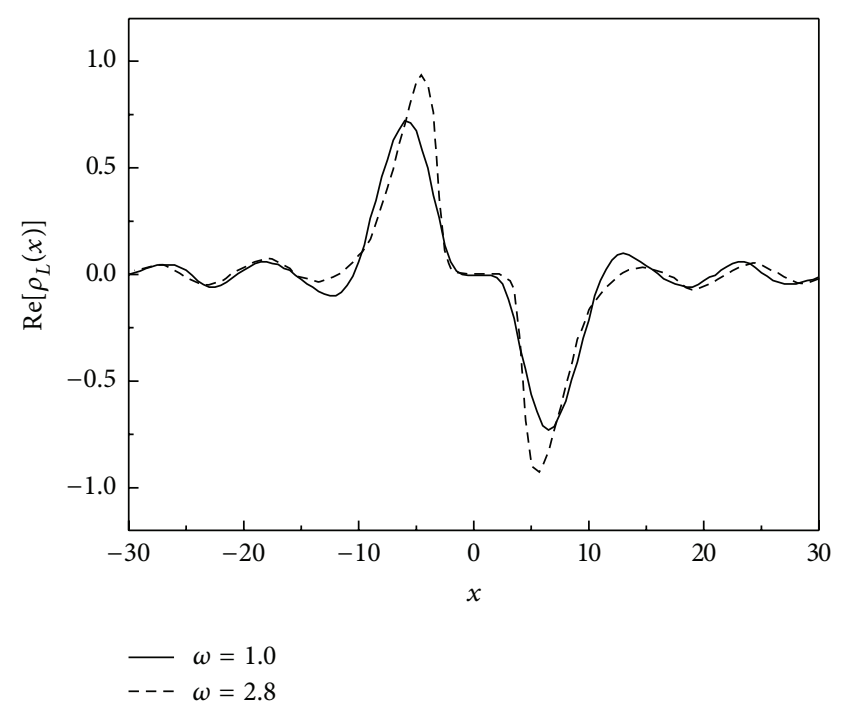

FIGURE 3: Distributions of the induced charge density (real part) by setting $\omega=1,2.8$.

At zero temperature, the diagonal matrix elements of conductance $G_{11}$ in Figure 5 show some positive and negative resonant peaks around the integral values of $\Delta$ with the increase of the Fermi energy; this corresponds to the step jump of the dc conductance of the QW, which is caused by the opening of additional quantum channels in the QW. This is a resonance related to the transverse energy levels of the QW. Moreover, there are other types of resonance related to the longitudinal motion of electrons in the QW. $G_{11}$ and $C$ (in Figure 6) show step-like and oscillatory behaviors between the two neighboring resonances, which corresponds to the opening of the next higher channel. Also the strength and frequency of oscillation increase with the frequency of the external field increasing. This oscillation may be caused by longitudinal resonant electron states that occur when the 


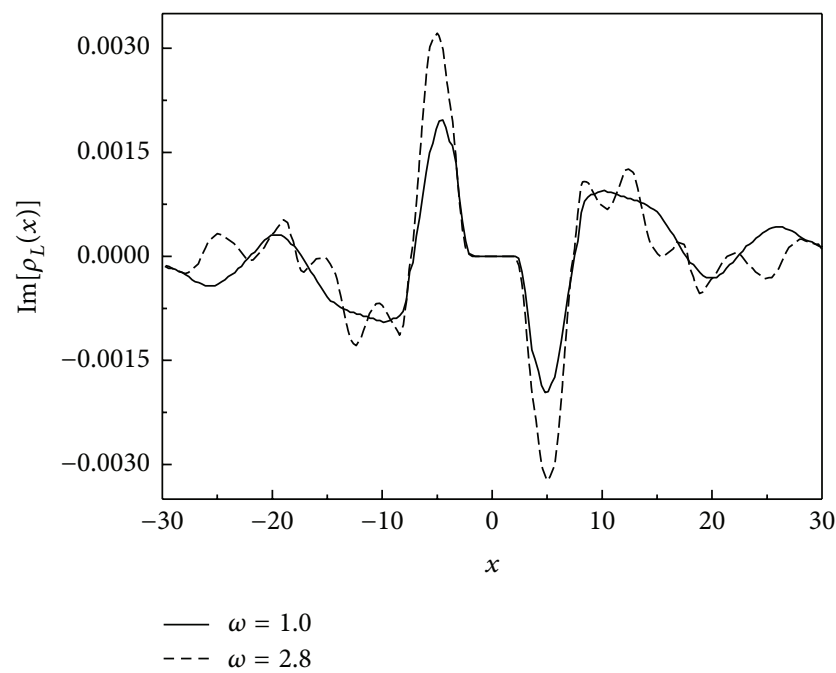

FIGURE 4: Distributions of the induced charge density (imaginary part) by setting $\omega=1,2.8$.

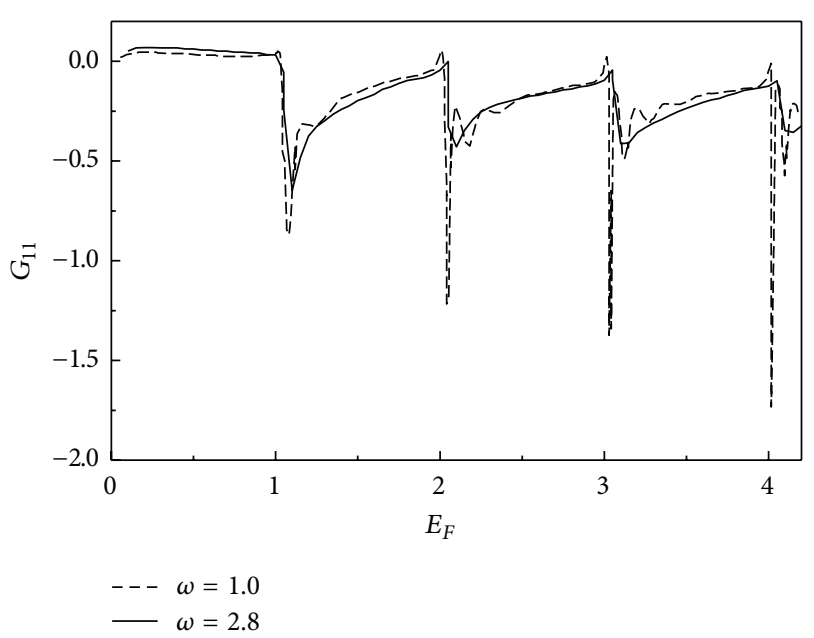

Figure 5: $G_{11}$ as the function of the Fermi energy for different frequencies, $\omega=1,2.8$.

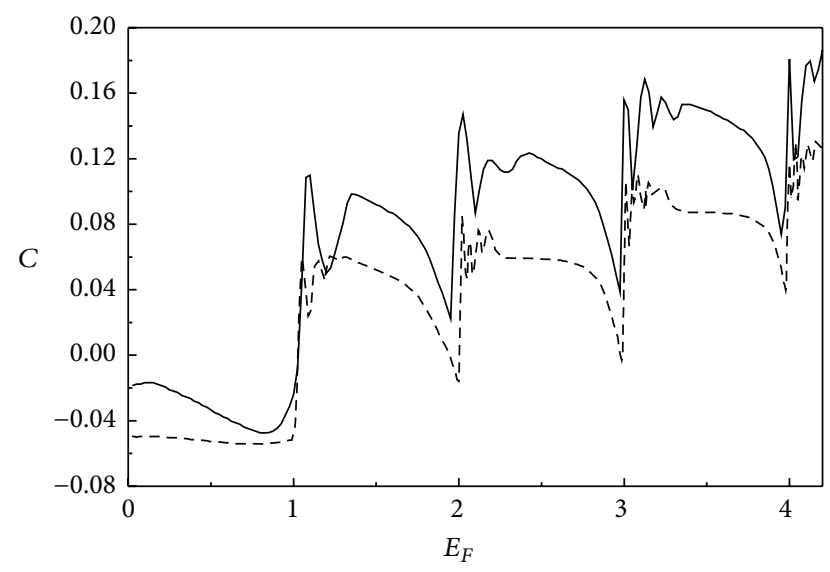

$$
-\omega=1.0
$$

Figure 6: $C$ as the function of the Fermi energy for different frequencies, $\omega=1,2.8$.

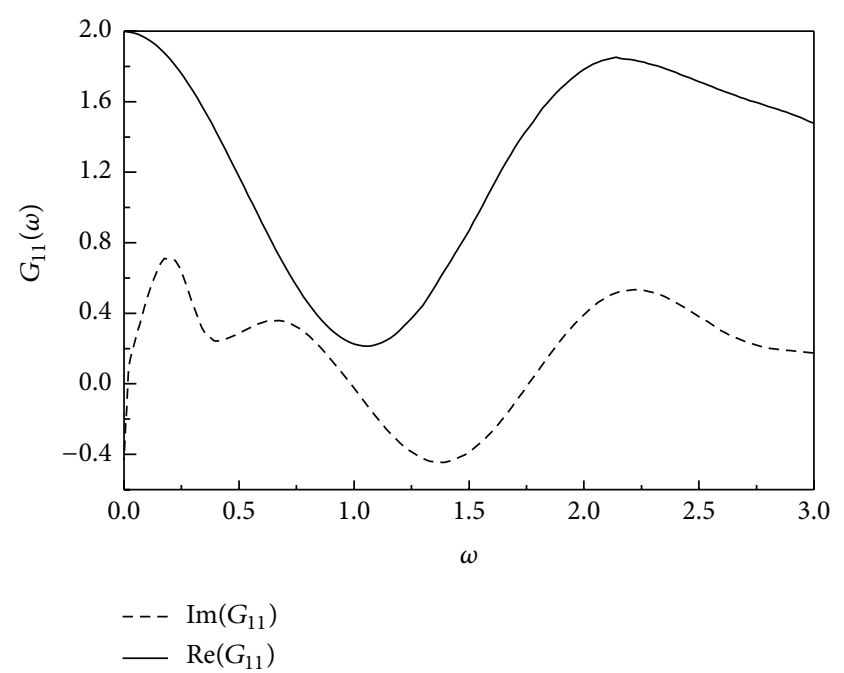

FIGURE 7: $G_{11}$ as the function of the frequency of the external field.

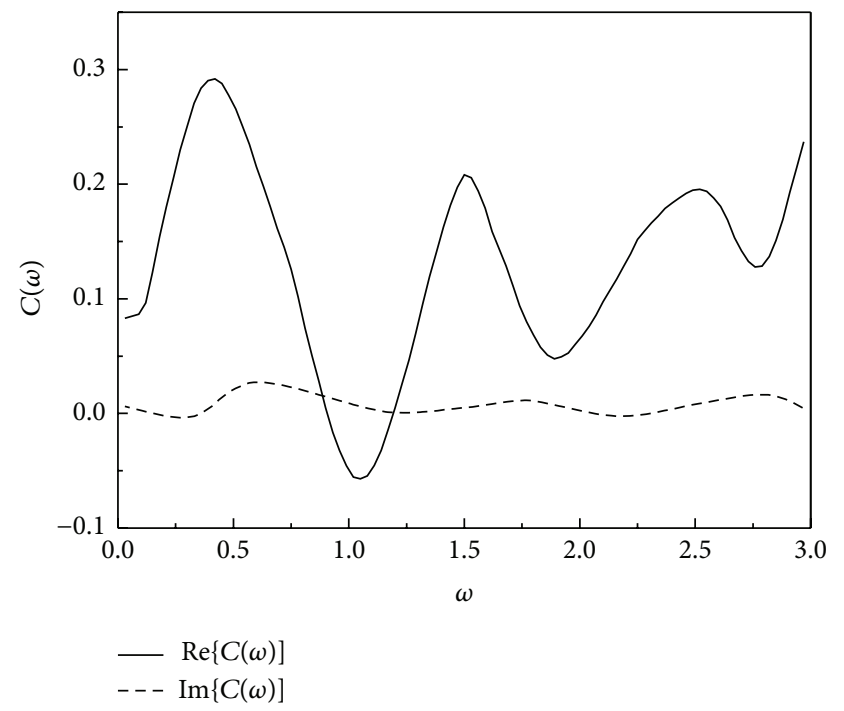

FIGURE 8: $C$ as the function of the frequency of the external field.

length of the QW is approximately equal to integer multiples of half longitudinal Fermi wavelength [15].

Figures 7 and 8 show $G_{11}(\omega)$ and $C(\omega)$ as functions of the frequency of external voltages by setting $E_{F}=2.4$, respectively, which is two times more than the ground energy. Towards this case, electrons through the second channel, the real part of $G_{11}(\omega)$ begins to decrease from two times conductance unit, and the real and imaginary parts $G_{11}(\omega)$ show irregular oscillatory behaviors. These indicate $e$ - $e$ interaction leads to the nonuniform distribution of current in transport system. Moreover, another important property is current phase. It should be pointed that the conductance is complex, in which the imaginary part refers to the phase difference between the current and voltage; this means that there are two different responses to the external voltage, inductivelike and capacitive-like, which depend on the plus sign and minus sign of the conductance matrix elements, respectively. 
As is well known, the plasmon excitations exist for interacting electron gas of bulk. It is significant to ask whether there is some quasiparticle excitation related to the collective motion of the electrons in the mesoscopic conductor. Reference [16] had indicated that there existed some excitations, such as plasmons, in mesoscopic conductor, and they had effect on the ac transport properties. Here, some peaks structure in curve of the real part of $C(\omega)$ are observed; we believe that these peaks are related to the plasmon excitation in the mesoscopic conductor.

\section{Conclusion}

In conclusion, we have presented the calculation of various physics quantities, such as the distributions of the internal potential and the induced charge density, the frequencydependent capacitance, and conductance of the QW. The induced charge is mainly distributed in the transition regions between the reservoirs. For the results of capacitance and conductance versus Fermi energy, some resonant peaks due to the opening of the next higher quantum channels and the oscillations related to the longitudinal resonant electron states of the QW are observed. The frequency-dependent conductance shows two different responses to the external voltage, inductive-like and capacitive-like, which depend on the plus sign and minus sign of the conductance matrix elements. The peaks structure of frequency-dependent capacitance is related to the plasmon-like excitation in mesoscopic conductor.

\section{Conflict of Interests}

The authors declare that there is no conflict of interests regarding the publication of this paper.

\section{Acknowledgments}

The authors gratefully acknowledge the support by the National Natural Science Foundation of China (Grant nos. 11304276,11404283 ) and the Natural Science Foundation of Guangdong Province, China (Grant nos. 2014A030307028, 2014A030307035).

\section{References}

[1] M. Buttiker, "Capacitance, admittance, and rectification properties of small conductors," Journal of Physics: Condensed Matter, vol. 5, no. 50, pp. 9361-9378, 1993.

[2] M. Büttiker, A. Prêtre, and H. Thomas, "Dynamic conductance and the scattering matrix of small conductors," Physical Review Letters, vol. 70, no. 26, pp. 4114-4117, 1993.

[3] A. Prêtre, H. Thomas, and M. Büttiker, "Dynamic admittance of mesoscopic conductors: Discrete-potential model," Physical Review B, vol. 54, no. 11, pp. 8130-8143, 1996.

[4] J. Wang, B. G. Wang, and H. Guo, "Quantum inductance and negative electrochemical capacitance at finite frequency in a two-plate quantum capacitor," Physical Review B, vol. 75, no. 15, Article ID 155336, 5 pages, 2007.
[5] Z. S. Ma, J. Wang, and H. Guo, "Weakly nonlinear ac response: theory and application," Physical Review B, vol. 59, no. 11, pp. 7575-7578, 1999.

[6] X.-A. Zhao and Y.-X. Chen, "Role of the internal potential in current conservation and gauge-invariant low-frequency dynamic mesoscopic transport," Physical Review B, vol. 64, Article ID 085326, 2001.

[7] V. Gasparian, T. Christen, and M. Büttiker, "Partial densities of states, scattering matrices, and Green's functions," Physical Review A-Atomic, Molecular, and Optical Physics, vol. 54, no. 5, article 4022, 1996.

[8] Y. M. Blanter and M. Büttiker, "Effect of interactions on the admittance of ballistic wires," Europhysics Letters, vol. 42, no. 5, pp. 535-540, 1998.

[9] V. A. Sablikov, S. V. Polyakov, and M. Büttiker, "Charging effects in a quantum wire with leads," Physical Review B, vol. 61, no. 20, pp. 13763-13773, 2000.

[10] V. A. Sablikov and B. S. Shchamkhalova, "Electron transport in a quantum wire with realistic Coulomb interaction," Physical Review B, vol. 58, no. 20, pp. 13847-13855, 1998.

[11] G. Cuniberti, M. Sassetti, and B. Kramer, "Ac conductance of a quantum wire with electron-electron interactions," Physical Review B: Condensed Matter and Materials Physics, vol. 57, no. 3, pp. 1515-1526, 1998.

[12] Q. r. Zheng, J. Wang, and H. Guo, "Low-frequency quantum transport in a three-probe mesoscopic conductor," Physical Review B, vol. 56, no. 19, pp. 12462-12468, 1997.

[13] J. Quan, Y. Tian, J. Zhang, and L. Shao, "Dynamic electron transport theory for multiprobe mesoscopic structures," Chinese Physics B, vol. 20, no. 7, Article ID 077201, 2011.

[14] B. Wang, J. Wang, and H. Guo, "Current partition: a nonequilibrium Green's function approach," Physical Review Letters, vol. 82, no. 2, article 398, 1999.

[15] H. Xu, "Theory of ballistic transport through a 3D-1D-3D quantum system," Physical Review B, vol. 48, no. 12, pp. 88788881, 1993.

[16] J. Quan, Y. X. Liu, and Y. B. Yu, "Dynamic response of the coherent parallel-plate capacitor to the external field," Acta Physica Sinica, vol. 59, no. 2, pp. 1237-1242, 2010. 

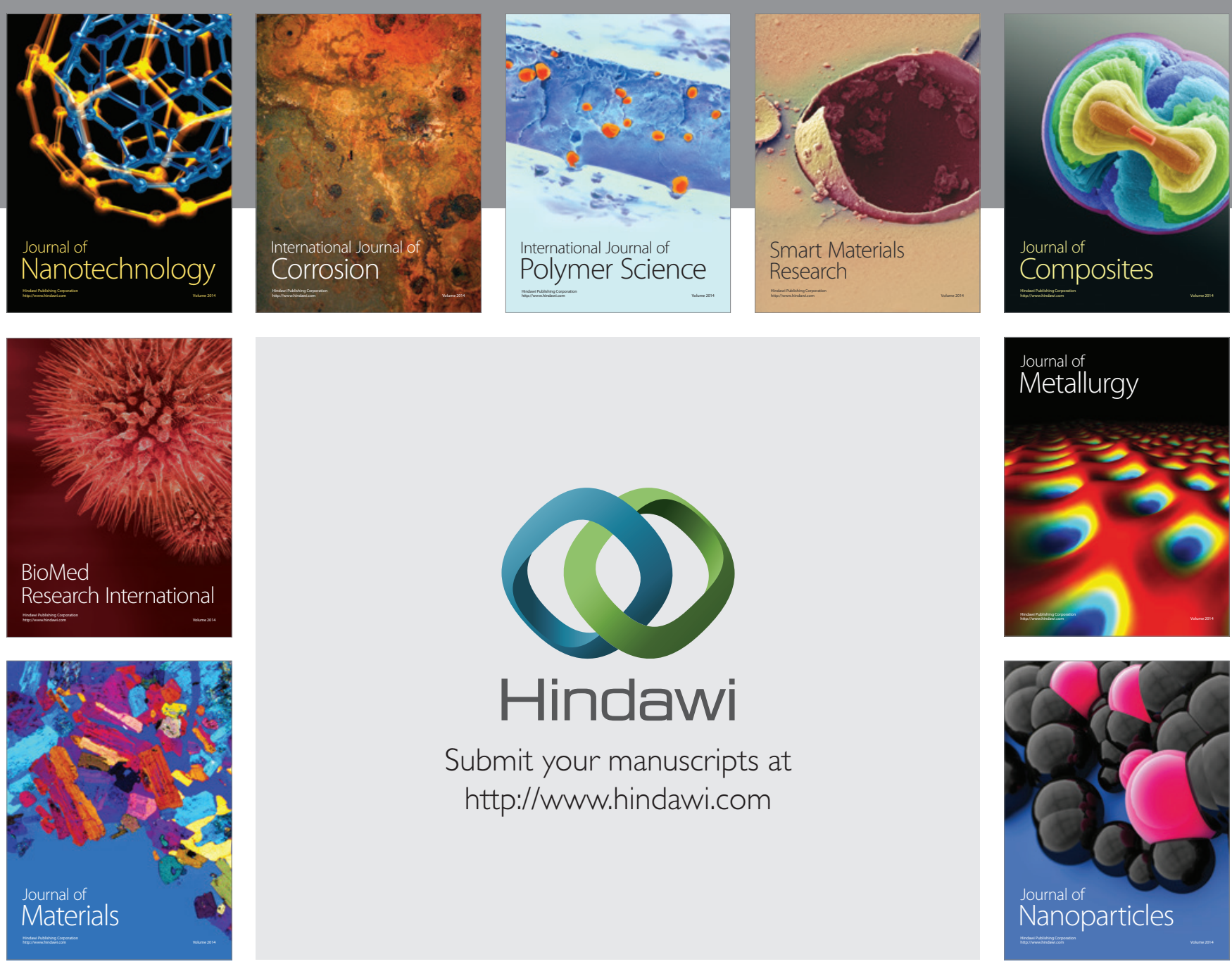

Submit your manuscripts at http://www.hindawi.com
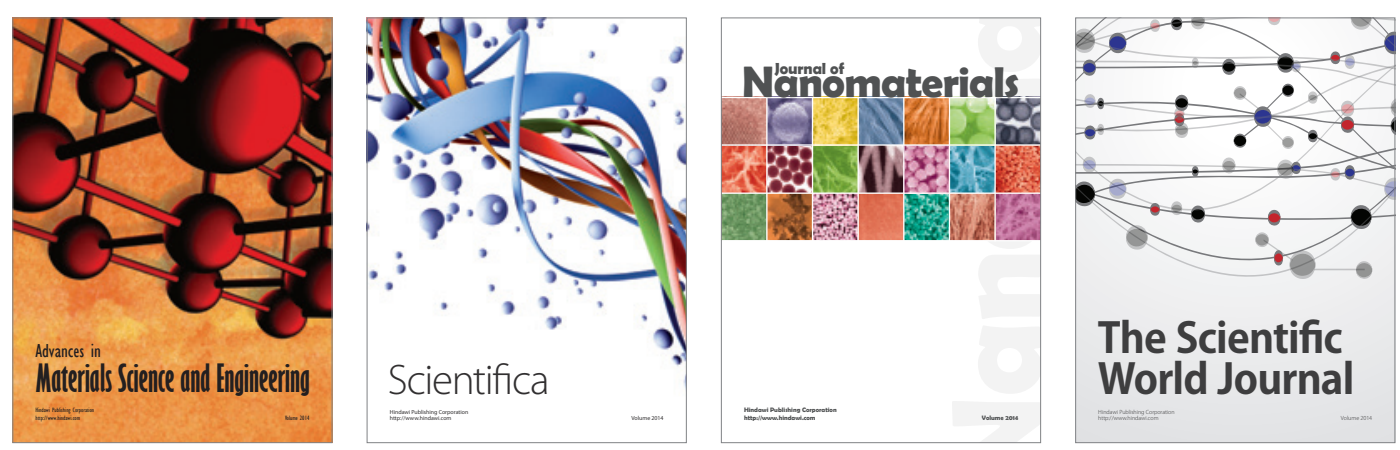

\section{The Scientific World Journal}
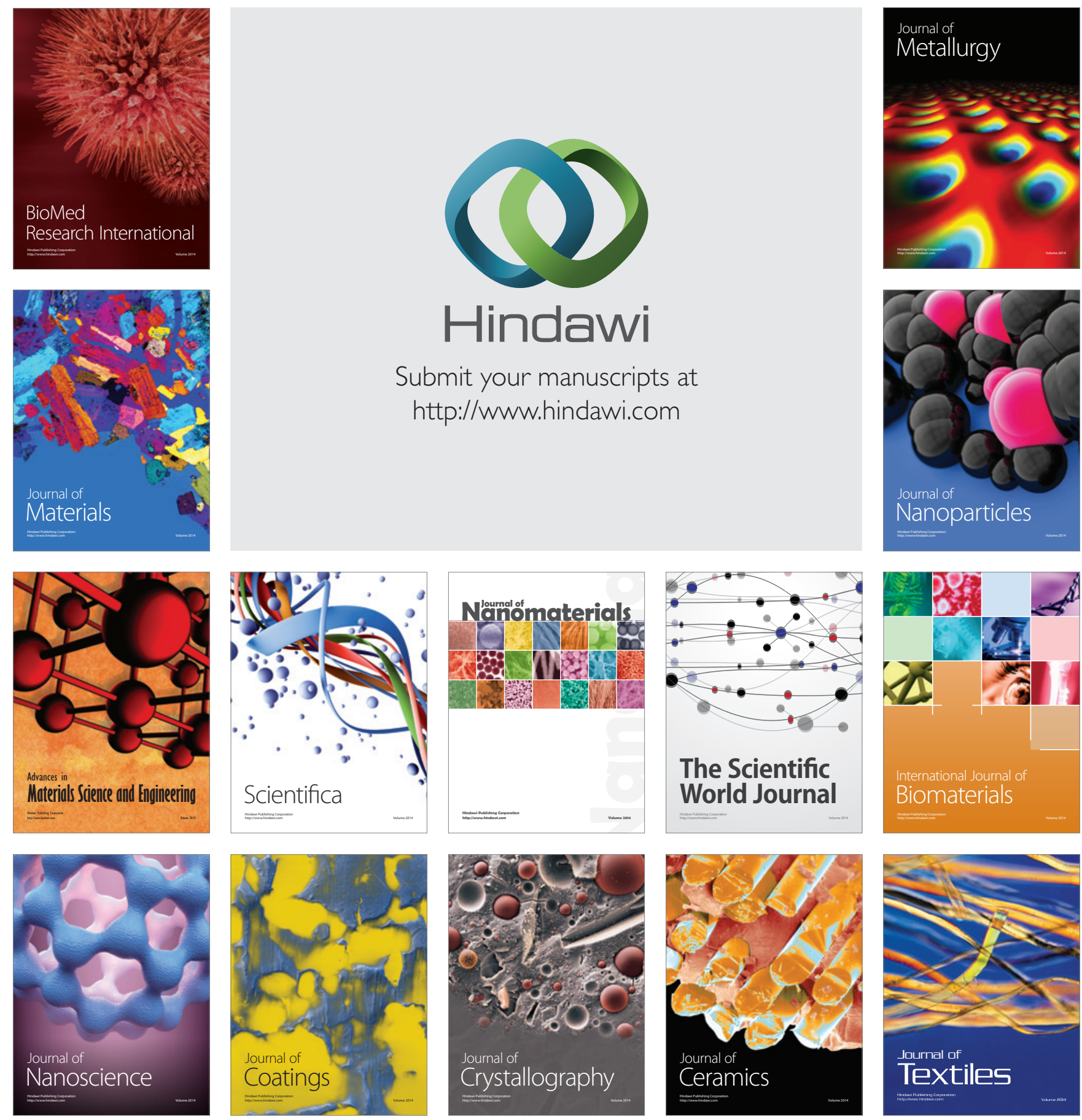